\title{
WILEY-VCH
}

\section{Magnetically Addressable Shape-memory and Stiffening in a Composite Elastomer}

Paolo Testa*, Robert W. Style, Jizhai Cui, Claire Donnelly, Elena V. Borisova, Peter M. Derlet, Eric R. Dufresne* and Laura J. Heyderman*

Paolo Testa, Dr. Jizhai Cui, Dr. Claire Donnelly, Prof. Laura J. Heyderman

Laboratory for Mesoscopic Systems

Department of Materials

ETH Zurich, 8093 Zurich, Switzerland

Email: paolo.testa@mat.ethz.ch, laura.heyderman@mat.ethz.ch

Paolo Testa, Dr. Robert W. Style, Prof. Eric. R. Dufresne

Laboratory for Soft and Living Materials

Department of Materials

ETH Zurich, 8093 Zurich, Switzerland

Email: eric.dufresne@mat.ethz.ch

Paolo Testa, Dr. Jizhai Cui, Dr. Claire Donnelly, Dr. Elena V. Borisova, Dr. Peter M. Derlet, Prof. Laura J. Heyderman

Paul Scherrer Institute

5232 Villigen, Switzerland

Keywords: soft matter, magneto-mechanical material, liquid inclusions, magneto-rheology, xray tomography

\begin{abstract}
With a specific stimulus, shape-memory materials can assume a temporary shape and subsequently recover their original shape, a functionality that renders them relevant for applications in fields such as biomedicine, aerospace and wearable electronics. Shapememory in polymers and composites is usually achieved by exploiting a thermal transition to program a temporary shape and subsequently recover the original shape. This may be problematic for heat sensitive environments, and when rapid and uniform heating is required. In this work, a soft magnetic shape-memory composite is produced by encasing liquid droplets of magneto-rheological fluid into a polydimethylsiloxane matrix. Under the influence of a magnetic field, this material undergoes an exceptional stiffening transition, with an almost 30-fold increase in shear modulus. Exploiting this transition, fast and fully reversible magnetic shape memory is demonstrated in three ways, by embossing, by simple shear and by unconstrained three-dimensional deformation. Using advanced synchrotron x-ray tomography
\end{abstract}




\section{WILEY-VCH}

techniques, the internal structure of the material is revealed, which can be correlated with the composite stiffening and shape-memory mechanism. This material concept, based on a simple emulsion process, can be extended to different fluids and elastomers, and can be manufactured with a wide range of methods.

\section{Main Text}

Shape-memory materials can assume a temporary shape through a programming step and can then recover their original shape in response to a specific stimulus. ${ }^{[1]}$ By encoding mechanical functionality in the material itself, rather than in the device, these materials allow for a reduction in the complexity of parts and an increase in energy efficiency. ${ }^{[2]}$ Compared to other classes of shape-memory materials, shape-memory polymers and composites are lightweight, inexpensive, can be made into parts using a variety of manufacturing technologies, and can have a broad range of properties. ${ }^{[3]}$ Thanks to these characteristics, they find applications in a variety of engineering areas including aerospace, ${ }^{[4]}$ wearable electronics ${ }^{[5]}$ and biomedicine. ${ }^{[6]}$ The design of shape-memory polymers is usually based on the combination of two distinct phases, a stable phase and a programmable phase. The programmable phase can undergo a reversible stiffening transition triggered by an external stimulus, while the stable phase remains unaffected. If the programmable phase is stiffer than the stable phase, the material can hold a temporary shape, while the inverse transition leads to the recovery of the original shape. ${ }^{[7]}$ Typically, the change in stiffness of the programmable phase is achieved through a thermally induced phase transition. ${ }^{[8]}$ Heating of the material, however, can be non-uniform and slow, since polymers are relatively good heat insulators. ${ }^{[9,10]}$ Additionally, the use of thermal stimuli greatly limits applications in heat sensitive environments such as the human body, as heat can harm living tissues. ${ }^{[11,12]}$ Therefore, when considering biomedical applications, careful tuning of the shape recovery temperature is required for safe employment of these materials since triggering temperatures 


\section{WILEY-VCH}

above body temperature can cause cell damage while, for activation temperatures below body temperature, the effect may be triggered prematurely. ${ }^{[13]}$

Here, we present an athermal, magnetically addressable shape-memory polymer composite, produced by dispersing droplets of a commercial magneto-rheological fluid in a cross-linked polydimethylsiloxane (PDMS) matrix, shown schematically in Figure 1a. Magneto-rheological fluids are able to undergo a substantial change at the millisecond time scale when subjected to a magnetic field, with mechanical properties such as viscosity, shear modulus and yield stress increasing by up to six orders of magnitude. ${ }^{[14,15]}$ By encasing the magneto-rheological fluid in an elastomeric matrix, we are able to manufacture a soft elastic composite with exceptional magneto-mechanical properties. The composite can undergo substantial stiffening when subjected to a magnetic field, with an exceptional increase in storage modulus of approximatively thirty times at a field of $600 \mathrm{mT}$ (see Figure $1 \mathrm{~b}$ and $2 \mathrm{e}$ ). In addition, the composite displays magnetically addressable shape-memory, where the polydimethylsiloxane matrix acts as the stable phase and the magnetic fluid as the programmable phase. An applied deformation can be programmed in the composite through the application of a magnetic field. The composite relaxes back to its original form in less than one second when the magnetic field is released, as shown in Figure 1c and in the Movie S1 available in the Supporting Information. The combination of various types of liquid inclusions with soft elastomeric matrices has been recently proposed as an effective way to create soft materials with novel functionalities, such as high electrical conductivity and temperature dependent color. ${ }^{[16-19]}$ We have extended this approach to our material, using instead a magneto-rheological fluid. This has a number of advantages compared to previously reported composites in which the magnetic particles are directly dispersed in the polymer matrix.${ }^{[20,21]}$ Due to the larger mobility of the particles when suspended in a liquid solvent, the alignment of particles that causes the mechanical property change is more effective, resulting in a larger stiffening effect. At the same time, the degradation of mechanical properties often 


\section{WILEY-VCH}

observed with soft composites with incorporated hard particles, such as embrittlement and

reduced durability, is avoided thanks to the liquid nature of the magnetic component. ${ }^{[22]}$

We dispersed magneto-rheological fluid droplets in PDMS prior to cross-linking at volume fractions, $\phi$, ranging from $0 \%$ to $40 \%$, as described in the Experimental Methods. In order to understand the effect of the magnetic field on the microstructure of the material, we used x-ray tomographic imaging to assess the internal structure of the composite at length scales from $\sim 3 \mu \mathrm{m}$ up to $1 \mathrm{~mm}$. In this range, we can resolve the shape and internal structure of the droplets as well as their arrangement in the elastic matrix (full tomographic reconstructions of the composite structure, with and without the applied field, are available as Movie S2 and S3 in the Supporting Information). A 3D rendering of the tomographic reconstruction for a composite with $\phi=40 \%$ at $B=0 \mathrm{mT}$ is shown in Figure 1a, with a single 2D slice shown in Figure 2a. The highly x-ray absorbing iron particles inside the fluid are visible (grey), while the x-ray transparent silicone and solvent, consisting of a water and glycerol mixture, are essentially indistinguishable from each other. The single iron particles in the magnetic fluid have sizes in the range of 1 to $6 \mu \mathrm{m}$ (see Supporting Information, Figure S1 and S2), which is at the spatial resolution limit of the tomographic imaging tool. Although the single particles are not resolvable, the general microparticle distribution can be assessed and, when the sample is not subjected to a magnetic field, both the droplet shape and internal particle arrangement show no preferential orientation (Figure 2b). On application of a magnetic field, the microstructure changes, with both the droplet elongating and the particles inside the droplets aligning in the direction of the field (Figure 2c). To verify the observed alignment, we performed Fourier analysis of the three-dimensional structure, and analyzed the angular dependence of the Fourier Transform intensity (for details of the analysis see Supporting Information). The angle $\alpha$, shown in Figure 2c, indicates a specific orientation relative to the direction of the magnetic field. When the magnetic field is applied, we see an 


\section{WILEY-VCH}

enhancement of the Fourier transform intensity at an orientation parallel to the field,

indicating that the magnetic particles inside the fluid droplets orient along the field direction (see Figure 2d for an azimuthal representation and Figure S5 for the Fourier transform spectra). This feature is prominent for several different wavelengths in the range 5-50 $\mu \mathrm{m}$ (see Figure S6), which are comparable to the size of the magnetic particles $(\sim 5 \mu \mathrm{m})$ and the magnetic fluid droplets ( 20 to $100 \mu \mathrm{m})$. We further quantified the alignment by analyzing the angular dependency of the Fourier transform intensity in the region between $\alpha=0$ and $\alpha=$ $\pi / 2$ and calculating the orientation order parameter $S$, that describes the average orientation of the structure with respect to the magnetic field direction (for details on the calculation see Supporting Information). $S$ can assume values between 0 , meaning no preferential orientation, and 1, representing complete ordering. The value of $S$ when no magnetic field is applied is small and negative, suggesting the structure has no preferential orientation. After the application of the magnetic field $S$ is equal to 0.54 , which implies a partial orientation of the structure along the field direction.

The alignment of the microstructure in the magnetic field direction observed with the tomographic analysis has a dramatic impact on the composite rheology. In order to determine the extent of this modification to the rheological properties, we measured the magnetorheological response of the material in shear using a parallel plate rheometer equipped with a magnetic field generator, with the field-direction perpendicular to the plate surface. Frequency sweep data for the base components and for two composites with volume fractions, $\phi=10 \%$ and 40\%, are available in the Supporting Information (Figure S8 and Figure S9). The magnetic field dependence of the storage modulus for these two different values of $\phi$ is shown in Figure 2e. At a relatively low volume fraction, $\phi=10 \%$, the storage modulus of the composite increases almost two-fold with application of a $1000 \mathrm{mT}$ field. At $\phi=40 \%$, the same magnetic field results in an almost 30 -fold increase in the storage modulus. These values 


\section{WILEY-VCH}

are remarkably high compared to previous results obtained for composites with magnetic particles dispersed directly in PDMS matrices where storage moduli increases of only 1.3-1.8 fold were reported at significantly higher iron contents. ${ }^{[23]}$ The dissipative behavior of the material is also affected by the magnetic field (Figure $2 \mathrm{f}$ and Figure $\mathrm{S} 9 \mathrm{~b}$ ), with the highest change in loss factor with field observed in a composite with $\phi=40 \%$ (Figure 2f). Indeed, the loss factor displays a total decrease of 50\% on application of a magnetic field of $1000 \mathrm{mT}$ for high frequencies (100 rad/s), and a two-fold increase at the same field for low frequencies (1 $\mathrm{rad} / \mathrm{s})$. This change in the loss factor with magnetic field is much more significant than that previously reported for composites with magnetic particles dispersed in polymer matrices, ${ }^{[24]}$ and is relevant for applications requiring adaptive vibration damping. ${ }^{[25]}$

We have seen how the magnetic field has a significant effect on the mechanical properties of the material. We additionally determined how the volume fraction of the magneto-rheological fluid, $\phi$, influences the spatial arrangement of the different phases in the composite and how this in turn affects the magneto-mechanical properties of the material. We therefore measured the mechanical properties of the composite for different $\phi$, with the storage modulus $G^{\prime}$ versus $\phi$ shown in Figure 3a. It can be seen that, at a field of $1000 \mathrm{mT}$, the storage modulus increases with $\phi$, with a steep increase above $\phi=20 \%$. This suggests a corresponding change in the spatial arrangement of the magnetic fluid droplets with increasing $\phi$, which we characterized with x-ray tomography (for details on the analysis see the Supporting Information). In particular, we quantified the connectivity of the magnetic fluid droplet network using an order parameter, $P$, which is a measure of the ratio of the volume of magnetorheological fluid in the largest droplet to the total volume of the magnetorheological fluid in the observed tomography region. As shown in Figure $3 \mathrm{~b}$ and c, the connectivity increases sharply at $\phi>20 \%$ and, at this volume fraction, the droplets of magnetic fluid form a fully connected network above the percolation threshold. ${ }^{[26]}$ This 


\section{WILEY-VCH}

confirms that the sharp change in mechanical properties is linked to the abrupt microstructural transformation. $^{[27]}$

We now demonstrate that the composite possesses a magnetically addressable shapememory. This effect was quantified via the application of the controlled shear stress and magnetic field protocol shown in Figure 4a, employing the same rheometer used for the mechanical characterization. This measurement is analogous to the actions performed on a strip of material, shown in Figure 4b and Movie S4 available in the Supporting Information. Initially, the composite is in its original stress-free state (Initial Stage 0). Upon application of external stress, the entire composite deforms into a temporary shape. Here, the elastomeric component of the composite develops a restoring stress, while the droplets in their liquid state remain stress-free (Stage 1). Upon application of a uniform magnetic field, the droplets solidify in a nearly stress-free state, while the continuous phase is unaffected (Stage 2). When the external stress is released, the restoring stress in the polymer network is counteracted by the stiffness of the droplet phase. If the yield stress of the droplet phase is much larger than the stresses in the polymer network, the polymer network achieves only a partial elastic recovery, in this case only $10 \%$, and the material retains a profile close to its programmed shape (Stage 3). When the magnetic field is removed, the droplets fluidize, allowing the elastomer to return to its original remembered shape (Stage 4). After this last step, a new cycle can be repeated, as the shape memory is completely re-writable.

In conclusion, we have presented a composite displaying unique magneto-mechanical properties, with an athermal, fast shape-memory and up to an almost 30-fold stiffening. Using advanced tomography methods, we determined the effect on these properties of the microstructural transformations induced by both the magnetic field and the volume fraction of magneto-rheological fluid. The fact that this shape-memory requires no heat, in conjunction with recent advances in magnetic control systems, ${ }^{[28]}$ opens up new possibilities for applications such as biomedical and wearable devices, which are operated in heat sensitive 


\section{WILEY-VCH}

environments. Based on a straightforward emulsion process, this material composite can not only be extended to different classes of polymer matrices and active fluids, but can also be manufactured with a wide range of methods, including casting, injection molding and additive manufacturing. ${ }^{[29]}$

\section{Experimental Section}

Fabrication of the composite: A water based magneto-rheological fluid (formulated on request, Liquids Research Limited) with $80 \%$ weight fraction of carbonyl iron microparticles was modified to avoid solvent evaporation by adding glycerol, resulting in a final fluid containing $66 \%$ weight fraction iron microparticles, $17 \%$ weight fraction of glycerol, and $17 \%$ weight fraction of water and stabilizers. At a relative humidity of approximately $40 \%$, drying was not observed over the course of one week, as expected from the equilibrium composition of the water-glycerol mixture. ${ }^{[30]}$ Polydimethylsiloxane was obtained by mixing different ratios of vinyl terminated polydimethylsiloxane (DMS-V31, Gelest inc.) with (2535\% methylhydrosiloxane)-dimethylsiloxane copolymer, trimethylsiloxane terminated (HMS301, Gelest Inc.), with the addition of platinum divinyl tetramethyldisiloxane catalyst (SIP6831.2, Gelest Inc.) according to the methodology found in Ref. ${ }^{[16]}$ to obtain the desired elastic modulus. To form the liquid precursor emulsion for the final composite, appropriate ratios of fluid and PDMS were added together with the surfactant molecule PEG-dimethicone (ES5612, DOW Corning) and stirred manually for 5 minutes. This results in a water-in-oil emulsion driven by the de-mixing of the water-based magneto-rheological fluid and the silicone precursors. The emulsion is stabilized by the surfactant during polymerization. The mixture was de-gassed in a vacuum chamber for an additional 5 minutes and left to cross-link overnight at room temperature. 


\section{WILEY-VCH}

\section{Supporting Information}

Supporting Information is available from the Wiley Online Library or from the author.

\section{Acknowledgements}

We thank Jens Heller and Jonathan Halter for their help with initial tomography trials for the tomography beamtime proposal, the Complex Materials group at the Department of Materials at ETH Zurich for the use of their rheometer, Marius Wagner and Christian Furrer for their help with the realization of the tomography holder, Valerio Scagnoli for help with the interpretation of the Fourier transform plots. We additionally thank Andrea Testa, Laura Maurel, Qin Xu, Nicolas Bain, Dominic Gerber, and Alba Sicher for their help with the experimental work and useful discussions. This work was funded by an ETH Research Grant (grant number ETH-48 17-1 'Tailored mesoscopic magneto-mechanical systems', awarded for a project proposed by P.T., P.M.D. and L.J.H.).

Received: ((will be filled in by the editorial staff))

Revised: ((will be filled in by the editorial staff)) Published online: ((will be filled in by the editorial staff))

\section{References}

[1] A. Lendlein, S. Kelch, Angew. Chemie Int. Ed. 2002, 41, 2034.

[2] W. M. Huang, Z. Ding, C. C. Wang, J. Wei, Y. Zhao, H. Purnawali, Mater. Today

2010, 13, 54 .

[3] W. Sokolowski, A. Metcalfe, S. Hayashi, L. Yahia, J. Raymond, Biomed. Mater. 2007, 2, S23.

[4] W. M. Sokolowski, A. B. Chmielewski, S. Hayashi, T. Yamada, in Proc. SPIE 3669, Smart Struct. Mater. (Ed.: Y. Bar-Cohen), 1999, pp. 179-185.

[5] M. Zarek, M. Layani, I. Cooperstein, E. Sachyani, D. Cohn, S. Magdassi, Adv. Mater. 2016, 28, 4166.

[6] A. Tonazzini, S. Mintchev, B. Schubert, B. Mazzolai, J. Shintake, D. Floreano, Adv. Mater. 2016, 28, 10142.

[7] M. D. Hager, S. Bode, C. Weber, U. S. Schubert, Prog. Polym. Sci. 2015, 49-50, 3.

[8] T. Xie, Nature 2010, 464, 267.

[9] W. Li, Y. Liu, J. Leng, J. Mater. Chem. A 2015, 3, 24532. 


\section{WILEY-VCH}

[10] J. M. Cuevas, J. Alonso, L. German, M. Iturrondobeitia, J. M. Laza, J. L. Vilas, L. M. León, Smart Mater. Struct. 2009, 18, 075003.

[11] C. Liu, H. Qin, P. T. Mather, J. Mater. Chem. 2007, 17, 1543.

[12] J. Ortega, D. Maitland, T. Wilson, W. Tsai, Ö. Savaş, D. Saloner, Ann. Biomed. Eng. 2007, 35, 1870.

[13] L. Yahia, in Shape Mem. Polym. Biomed. Appl., Elsevier, 2015, pp. 3-8.

[14] S. Elizabeth Premalatha, R. Chokkalingam, M. Mahendran, Am. J. Polym. Sci. 2012, 2,50 .

[15] H. M. Laun, C. Gabriel, Rheol. Acta 2007, 46, 665.

[16] R. W. Style, R. Boltyanskiy, B. Allen, K. E. Jensen, H. P. Foote, J. S. Wettlaufer, E. R. Dufresne, Nat. Phys. 2015, 11, 82.

[17] N. Kazem, M. D. Bartlett, C. Majidi, Adv. Mater. 2018, 30, 1706594.

[18] D. Doblas, J. Hubertus, T. Kister, T. Kraus, Adv. Mater. 2018, 30, 1803159.

[19] J. A. Jackson, M. C. Messner, N. A. Dudukovic, W. L. Smith, L. Bekker, B. Moran, A. M. Golobic, A. J. Pascall, E. B. Duoss, K. J. Loh, C. M. Spadaccini, Sci. Adv. 2018, 4, eaau6419.

[20] G. Filipcsei, I. Csetneki, A. Szilágyi, M. Zrínyi, Adv. Polym. Sci. 2007, 206, 137.

[21] Y. Li, J. Li, W. Li, H. Du, Smart Mater. Struct. 2014, 23, 123001.

[22] M. D. Bartlett, A. Fassler, N. Kazem, E. J. Markvicka, P. Mandal, C. Majidi, $A d v$. Mater. 2016, 28, 3726

[23] W. H. Li, M. Nakano, Smart Mater. Struct. 2013, 22, 055035.

[24] M. Kallio, Doctoral Thesis, Tampere University of Technology, 2005.

[25] V. S. Molchanov, G. V. Stepanov, V. G. Vasiliev, E. Y. Kramarenko, A. R. Khokhlov, Z. D. Xu, Y. Q. Guo, Macromol. Mater. Eng. 2014, 299, 1116.

[26] M. D. Rintoul, S. Torquato, J. Phys. A. Math. Gen. 1997, 30, L585. 


\section{WILEY-VCH}

[27] A. K. Kota, B. H. Cipriano, M. K. Duesterberg, A. L. Gershon, D. Powell, S. R.

Raghavan, H. A. Bruck, Macromolecules 2007, 40, 7400.

[28] J. Rahmer, C. Stehning, B. Gleich, Sci. Robot. 2017, 2, 1.

[29] Y. Kim, H. Yuk, R. Zhao, S. A. Chester, X. Zhao, Nature 2018, 558, 274.

[30] Physical Properties of Glycerol and Its Solutions, Glycerine Producers' Association, New York, 1953 
a

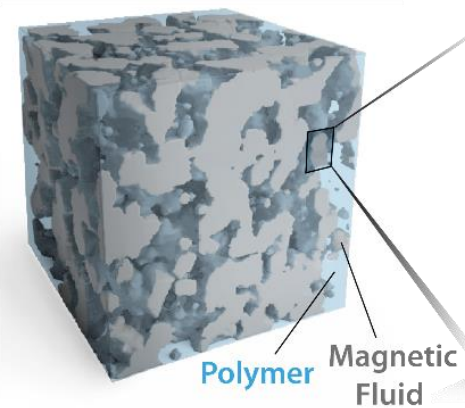

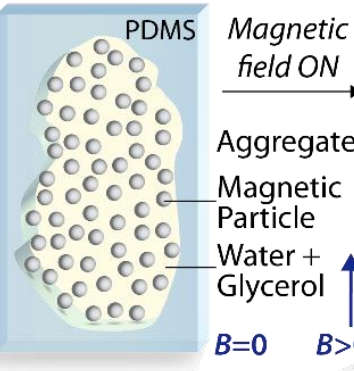

b

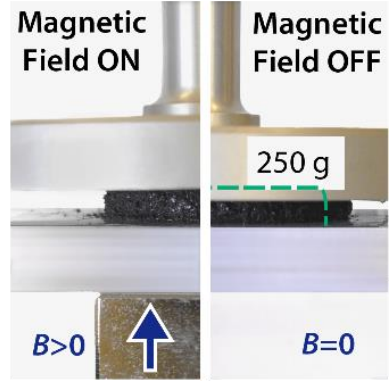

C

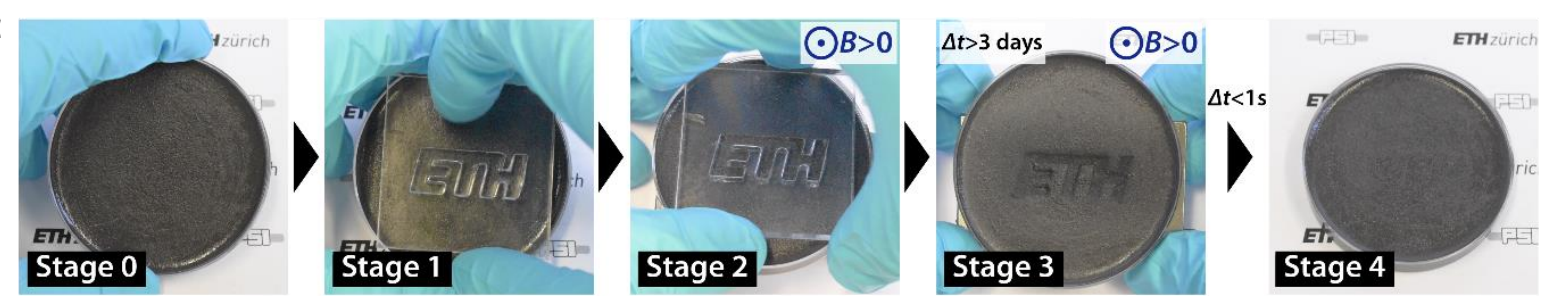

Figure 1. Magnetically-addressable stiffening and shape-memory in a polymer/magnetorheological fluid composite a) Three-dimensional reconstruction of the internal structure of the material obtained from tomography data, providing a 3D visualization of the two different phases in the composite (cube edge $=390 \mu \mathrm{m}$ ). The effect of the magnetic field on the internal structure of the magnetic phase in a droplet is schematically shown. With no magnetic field applied, the carbonyl iron microparticles are uniformly dispersed in the carrier solvent, consisting of water and glycerol, and the droplet is in a liquid state. After the application of a magnetic field, the particles rearrange to form elongated aggregates, resulting in a stiffness increase of several orders of magnitude in the magnetic fluid. b) Experimental demonstration of the influence of the magnetic field on the stiffness of the material. A disk of material is able to carry a weight of $250 \mathrm{~g}$ when subjected to a magnetic field from a permanent magnet (left), while it becomes soft and compliant under the same weight when the magnet is removed (right). c) Embossing a disk of material demonstrating magnetic shape-memory. The material can be programmed to retain a certain shape in a magnetic field, and returns to the original shape after removal of the field. A disk of material (initial Stage 0) is embossed using a stamp with a defined profile (Stage 1) and subsequently an out-of-plane magnetic field is applied with a permanent magnet (Stage 2). The material retains the programmed shape for several days (Stage 3) until the magnetic field is removed, after which it returns to the original shape in less than 1 second (Stage 4). Images are taken from Movie S1 available in the Supporting Information. 


\section{WILEY-VCH}
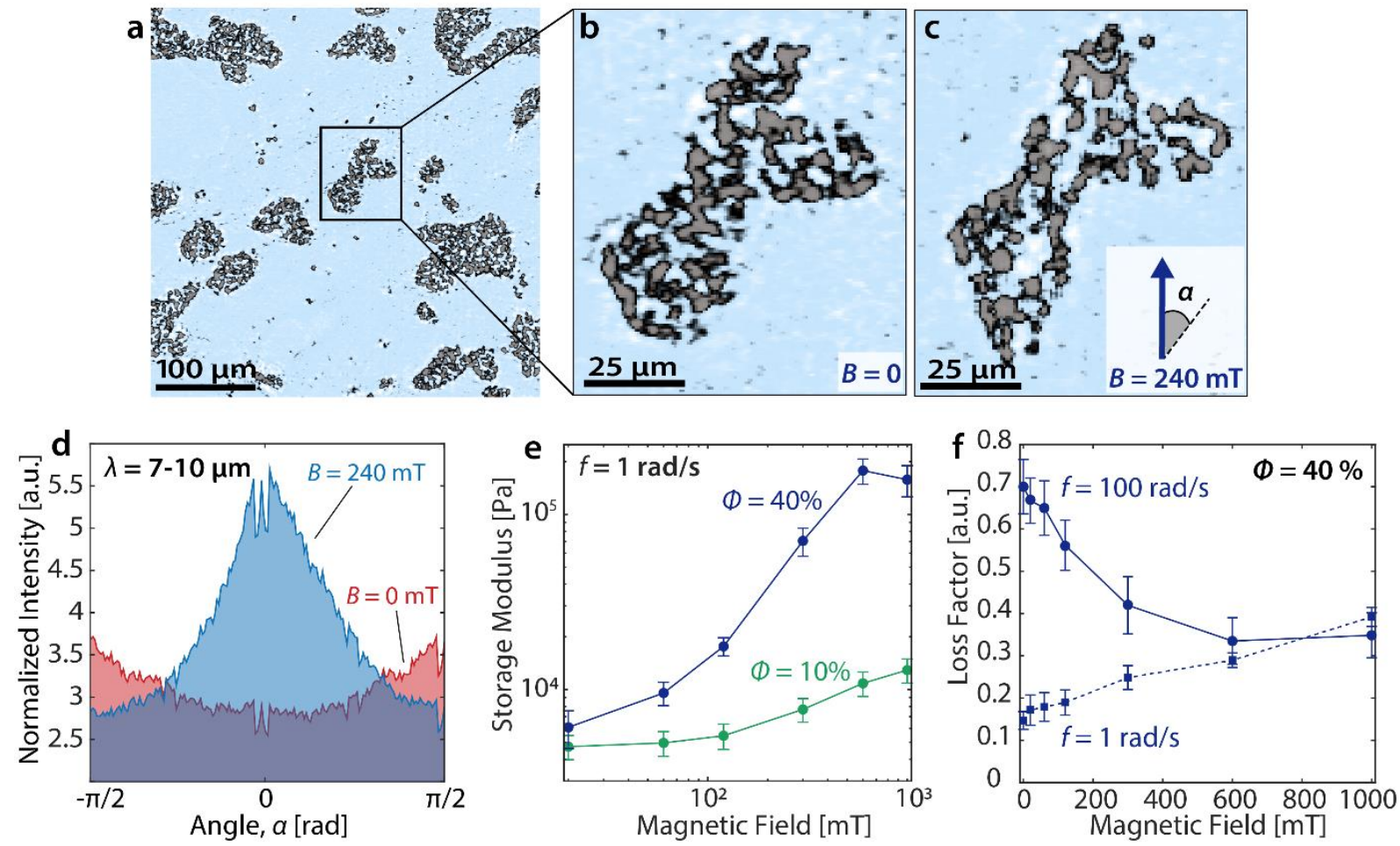

Figure 2: Effect of the magnetic field on the structure and properties of the composite elastomer. a) Two-dimensional slice of an x-ray absorption tomogram, obtained for a composite with $\phi=40 \%$ and with no magnetic field applied. The iron particles, in grey, can be distinguished from the rest of the components, in light blue. b) Close-up view of a single aggregate from the slice in panel a. c) The same aggregate in b) after the application of a magnetic field of $240 \mathrm{mT}$, demonstrating the re-arrangement of the iron particles with droplet elongation in the direction of the applied field. d) Azimuthal plot of the normalized, average Fast Fourier Transform intensity for complete tomographic stacks of the same sample taken at fields of $B=0 \mathrm{mT}$ and $B=240 \mathrm{mT}$. The plot refers to data in a wavelength range, $\lambda=7$ $10 \mu \mathrm{m}$, corresponding to the length scale of the magnetic particles in the fluid. The emergence of a peak after the application of the magnetic field in the vicinity of $\alpha=0$ indicates that the magnetic particles inside the fluid droplets orient along the field direction. e) Magnetic fieldinduced change in the storage modulus for $\phi=10 \%$ and $\phi=40 \%$. Higher stiffening is observed for larger $\phi . \mathrm{f}$ ) Change in the loss factor at frequencies of $f=1 \mathrm{rad} / \mathrm{s}$ and $f=100$ $\mathrm{rad} / \mathrm{s}$ with magnetic field for a composite with $\phi=40 \%$. 

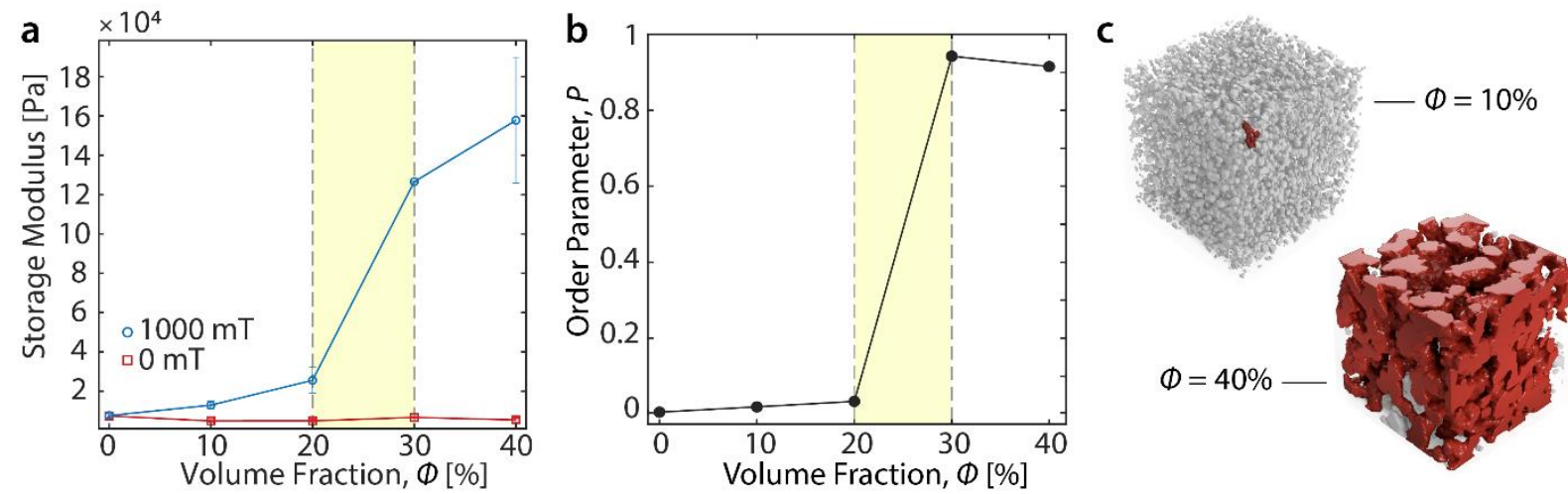

Figure 3: Effect of the fluid volume fraction on the stiffness and structure of the

material. a) Dependence of storage modulus on droplet volume fraction, $\phi$. The blue (red) points are data for an applied magnetic field of $1000 \mathrm{mT}(0 \mathrm{mT})$. A sharp increase in the storage modulus is observed at $\phi>20 \%$, corresponding to a change in the spatial arrangement of the phases. b) Increase of connectivity with $\phi$, quantified by the order parameter, $P$. The sharp increase of the value of $P$ at $\phi>20 \%$ indicates the onset of a fully connected magnetic fluid network, which in turn results in the sharp change in mechanical properties shown in panel a. c) Three-dimensional reconstruction of the internal structure of the material at $\phi=10 \%$ and $40 \%$ (cube edge $=390 \mu \mathrm{m}$ ). Highlighted in red is the biggest aggregate in the analyzed volume, which visually shows that the network of magnetic fluid is completely connected.
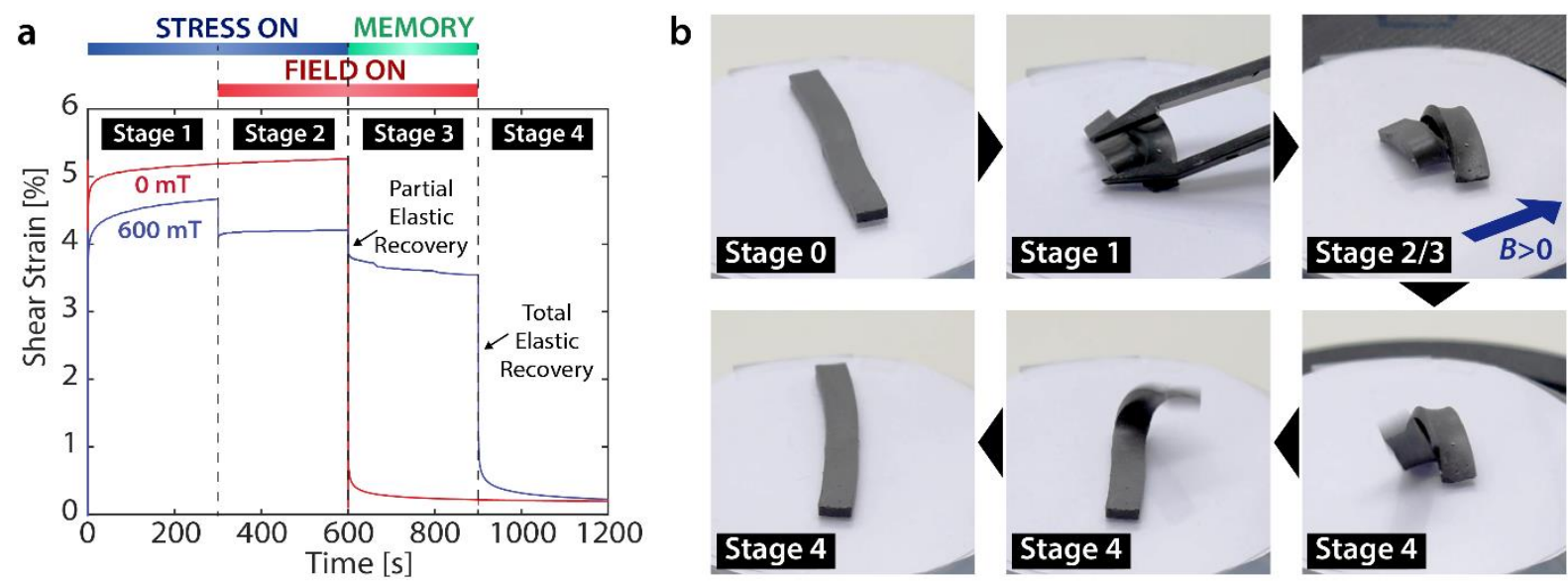

Figure 4: Magnetic shape-memory. a) Stepwise stress experiment performed in the absence (blue curve) and the presence (red curve) of a magnetic field. In zero field, the material recovers its original state once the stress is removed (Stage 2 to Stage 3). When a magnetic field of $600 \mathrm{mT}$ is applied, the material retains almost all of the deformation (Stage 3), and recovers the original shape once the field is removed (Stage 4). b) Experimental demonstration of magnetic shape-memory in an unconstrained strip deformed in three dimensions. A $4 \mathrm{~cm}$-long strip can be deformed into an arbitrary shape, which is stabilized with a uniform magnetic field applied using an arrangement of permanent magnets (Stage 2/3). This programmed shape is retained until the magnetic field is removed and the strip returns to its remembered shape in a few seconds (Stage 4). The images are taken from Movie S4 available in the Supporting Information. 


\section{Click here to access/download Supporting Information Supplnfo_adma201900561.docx \\ Supporting Information}


Click here to access/download Production Data S1_embossing_480p_DSC072.mp4 
Click here to access/download

\section{Production Data}

S2_Full_Stack_B0.mp4 
Click here to access/download Production Data S3_Full_Stack_B240.mp4 
Click here to access/download Production Data S4_memory_480p_DSC139_edit.mp4 
Click here to access/download
Production Data
Fig1.png
Fig Click here to access/download
Production Data
Fig1.png
Fig Click here to access/download
Production Data
Fig1.png
Fig

.

.

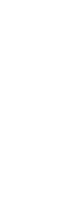 \\ Fig1.p
}

for

(1)

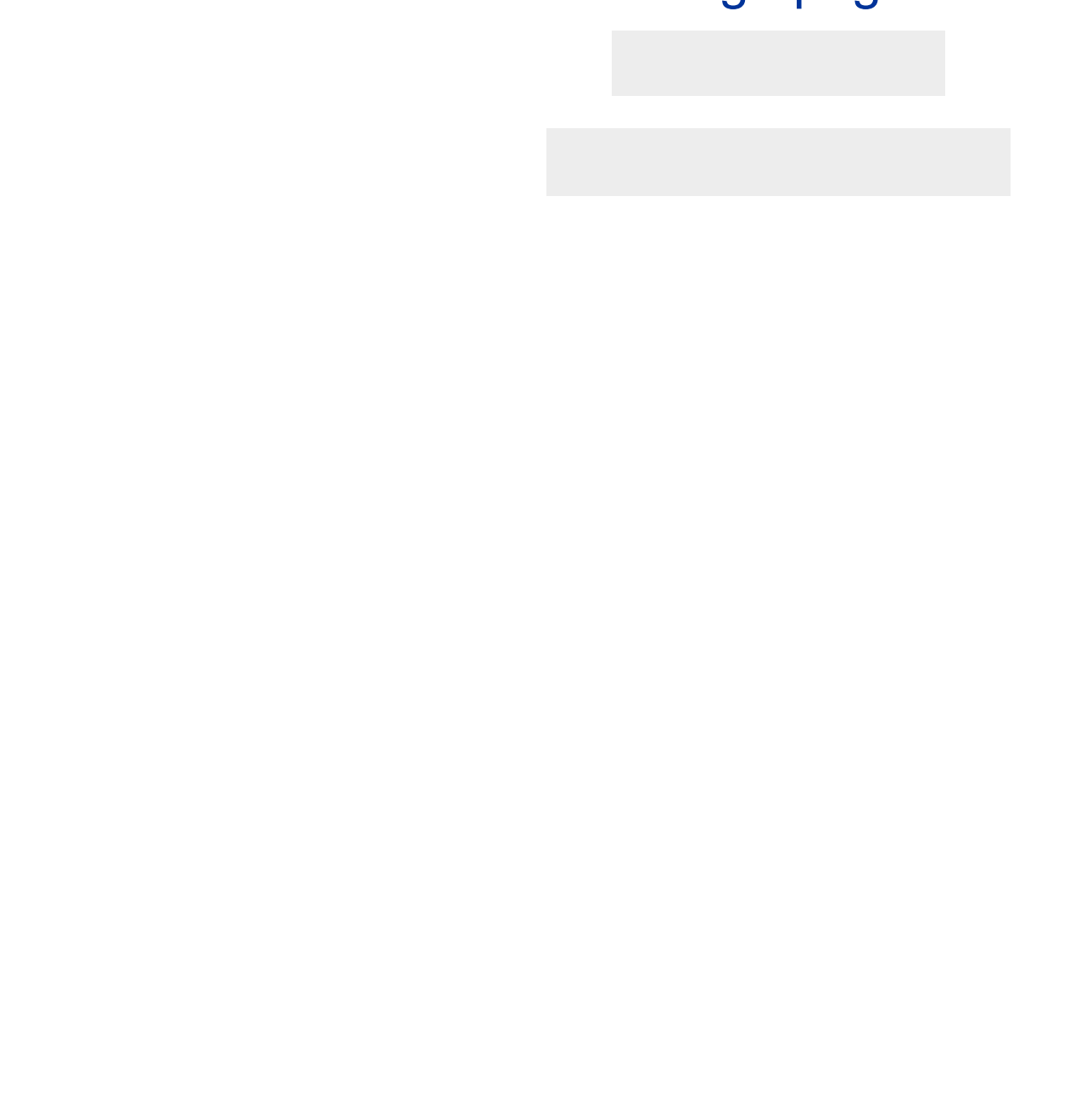


Production Data Fig2.png

\section{Click here to access/download}




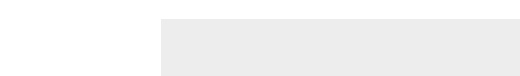

.

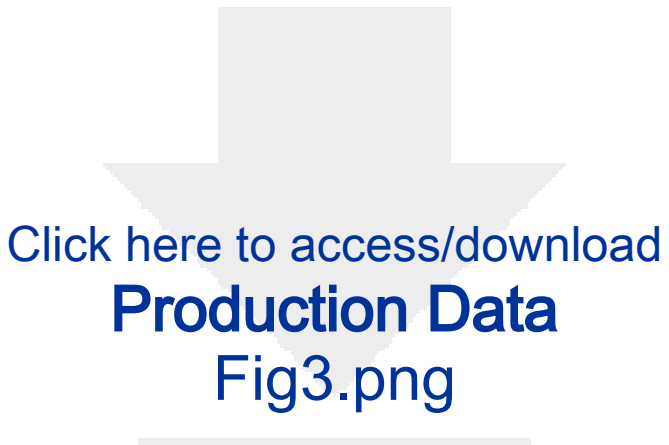

(1) 
Production Data Fig4.png

\section{Click here to access/download}


Click here to access/download

Production Data ToC.png 
Click here to access/download Production Data ToC_adma201900561.docx 
Fig1 vector
Click here to access/download
Production Data Fig1.ai

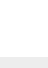

a
政

.

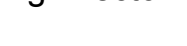


Click here to access/download Production Data Fig2.ai 
Production Data
Fig3.ai

Click here to access/downlc
Production Data
Fig3.ai Click here to access/dowı
Production Data
Fig3.ai

Fig3 vector

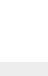

Fig3 vector

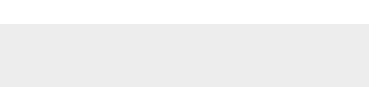

-

and

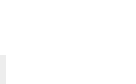

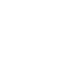

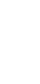
(n) 
ToC Figure vector

Click here to access/download Production Data ToC.ai 